This is the post-print version of the final paper published in Computers in Industry, 64(7), 785-797, 2013. The published article is available from http://www.sciencedirect.com/science/article/pii/S016636151300095X. Changes resulting from the publishing process, such as peer review, editing, corrections, structural formatting, and other quality control mechanisms may not be reflected in this document. Changes may have been made to this work since it was submitted for publication. Copyright @ 2013 Elsevier B.V.

\title{
Instant 3D Design Concept Generation and Visualisation by Real-Time Hand Gesture Recognition
}

\author{
Jinsheng Kang, Kang Zhong, Shengfeng Qin, Hongan Wang, David Wright
}

\begin{abstract}
:
$3 \mathrm{D}$ conceptual design is a creative process. While the designers are in the inspirational mode, they want to quickly express the design ideas in $3 \mathrm{D}$ and visualize it in real time without obstacle. In an exploration of a new, natural and more user friendly interface to assist rapid 3D conceptual generation and visualization, a prototype system of hand gesture and motion capture based user interface has been proposed and implemented. In this paper, we present the framework and components of this real time 3D conceptual generation and visualization system. Hand gestures were designed with the consideration of ease to use and the suitability for real time continuous recognition. Rule-based intelligent menu/icons were designed which changes the prompts according to the design process and expected operations. Real time hand gesture recognition was realised by skeleton model based template matching, and the use of Hidden Markov Models (HMMs). The recognised hand gestures become command script in OpenSCAD environment, and the 3D design concept was instantly generated and displayed on the screen. A case study was conducted for the initial evaluation of the system.
\end{abstract}

\section{Keywords:}

3D conceptual design, Hand gesture, Motion capture, Computer Aided Design, Concept Generation and Visualisation. 

1 Introduction

3D design concept generation and visualisation is an important part in conceptual design, in order to express, validate and evaluate the design ideas in 3D space. With the rapid development of computer hardware, especially CPU and GPU, 3D modelling in current computer-aided design (CAD) system becomes more and more powerful. However, the user interaction method of current CAD software has changed little, and become one of the bottlenecks of rapid 3D conceptual design. The keyboard and mouse based interface makes current CAD systems difficult to use for rapid 3D conceptual design generation and exploration. This issue has two aspects. First, conceptual design is a creative process, and a combination of art and engineering. While designers are in the creative design mode with inspiration, they want to quickly express the design ideas in 3D without obstacle. However, operating current CAD software to produce 3D conceptual design is time consuming and sometimes a burden - especially in the concept stages where designs change frequently. Secondly, the modelling with 3D CAD software requires complete, concrete and precise design information as input, which is often not available at the conceptual design stage. Therefore, it is difficult for designers to create rapidly 3D concept shapes, and change designs in current CAD systems. Zheng et al [1] discussed the drawbacks of CAD systems in 3D concept design and the need for improvement on user interface. Robertson and Radcliffe investigated the influences of current CAD systems on circumscribed thinking, premature design fixation, and bounded ideation [2].

Due to these drawbacks of traditional CAD systems, attempts have been made to allow designers to model 3D objects using more natural and efficient interaction mechanisms. The 3-Draw system [3] used a pair of hand-held, 6 DOF input devices to let the designer sketch out ideas in 3D. Recently, the system developed by Chen et al. [4] allowed users to design 3D objects by sketching in mid air the wireframes of the objects with an easily-tracked wand. The real time 3D positions of the strokes of the wand were captured by a camera, and the wireframes and the surfaces being developed were displayed on the PC screen to guide the user to draw more and more complex objects. However, users need to have a very good spatial imagination to use these systems, and wireframe modelling inherited the difficulties in surface editing and generating complex shapes. In a recently developed "Shape-It-Up" system, creative expression of 3D shapes in conceptual design was realised through the naturalistic integration of hand gestures, using a modelling scheme of intelligent generalized cylinders [5]. 
Attempts have also been made to integrate virtual reality with CAD, and develop some VR-based CAD systems. This type of system allows designers to create and modify CAD models using 3D devices in the virtual environment. DesignSpace [6], as an experimental testbed developed by Stanford University, aimed to offer the designer better access to the product models. From DesignSpace, several projects were developed, such as TalkingGlove, CutPlane, VirtualHand, VirtualGrasp, and TeleSign, which allow conceptual designers to create simple surface and solid geometric shapes using voice and gestures in mechanical design. COVIRDS (Conceptual Virtual Design System) [7, 8] provided a 3D virtual environment for the designer to model mechanical and electro-mechanical components. The system uses voice commands and hand motion/action as input modes for the designer to create 3D designs. Virtual hands have been modelled and integrated into virtual production line in an attempt to design better human-machine interactions [9]. Zheng et al [1] developed a desktop CAD system, which used a CyberGlove as an input device, for facilitating conceptual design. In this system, designers are allowed to use some simple gestures to conduct various geometric shape operations instead of depending solely on keyboard and mouse. Fuge et al [10] developed a system for directly creating and modifying free form surfaces through 3D hand gestures wearing a glove with coloured fingertips, in an augmented reality environment. "Mockup Builder" is a newly developed semi-immersive environment for users using 3D hand gestures to sketch, create and manipulate 3D shapes in conceptual design [11]. In these VR/AR based systems, the hand gestures defined were too simple to accomplish the whole design process alone. In other words, these systems did not focus mainly on hand gestures as interaction methods.

Hand gesture based interaction in 3D space is considered as an intuitive way of interacting with 3D objects [12, 13]. For example, the GIVEN [14] toolkit (Gesture based Interactions in Virtual Environments) focused on collision detection between the user's hand and the object being manipulated and defined a gestural language based on this interaction. Hand gestures have been used to describe 3D objects [15] and to control 3D character animation [16]. PolyShop [17] environment allowed the use of both hands for various tasks in a VE. With the development of hand gesture recognition in recent years, researchers $[18,19,20]$ have paid attention to generating $3 \mathrm{D}$ models based on hand gesture and motion. An early effort by Shih and Shih [21] used gesture modelling to simulate architectural space and objects. Hand gestures designed by sign language grammar such as nouns or verbs are used to model 3D buildings. However, these gestures are too complicated for 
designers to remember and to use at the early conceptual design stages, and it is difficult to recognize them in real time. An alternative technique was to move a stylus held by one hand to perform 3D design, as in our previous work using hand motions to create large-sized free-from surface models [22], physical models [23] and architectural models [24].

In view of previous research, although various efforts have been made, the improvement of the user interface for rapid 3D conceptual design still remains as a great challenge. Recently, hand gesture as a natural way to interact with computers and to control domestic appliances has gained great attention both from academia and industry, and is considered as a new trend for the future. In the meantime, the use of motion capture system is increasingly popular, given the fact that more and more easy-to-use motion capture systems at affordable price are available, such as Kinect. Based on this background and our previous research on using hand motions for design, the objective of the current research is to investigate the feasibility of a hand gesture and motion capture-based user interface to allow designers to interactively realise rapid 3D concept design in real time. Although motion capture system has the capability for real time computer animation, this is the pioneer research on using motion capture system for design application in real time. The robustness of algorithms used in the system in real time hand gesture recognition, real time data transfer and transform, as well as real time 3D object generation and display, are also to be examined.

\section{System and methods}

\subsection{System framework}

The framework of the whole system is shown in Fig. 1, which consists of three parts: a commercial motion capture system, a real time hand gesture recognition engine, and a customized 3D design environment. In this 3D conceptual design system, the designer can perform a series of hand gestures to create, manipulate, and merge 3D solids. The designer's hand motion is captured by a motion-capturing system, and then sent to the hand gesture recognition engine as input. The recognition engine processes and classifies the input motion data in real time. The results of recognition are converted into a series of detailed commands for 3D conceptual design, and the outcome of each command is displayed in the 3D design environment in WYSIWYG style. 


\subsection{Hand motion capture}

A three-dimensional optical motion capture system from Motion Analysis ${ }^{\mathrm{TM}}$ is used to capture the designer's hand motion. Seven Eagle digital cameras are located circularly to acquire hand motion date in a rough volume of $2 \mathrm{~m} \times 3 \mathrm{~m} \times 1.5 \mathrm{~m}$, as shown in Fig. 2. The locations of the cameras and the size of the capture volume can be adjusted depending on the requirement. For hand motion capture, small reflective markers ( $5 \mathrm{~mm}$ in diameter) are attached to the designer's hands, so the cameras are located close to the user in order to capture the markers easier and more accurately. According to the discussion in the previous section on hand skeleton model, reflective markers are placed at the closest positions to corresponding hand joints, but not exactly on the joints in order to reduce the unnecessary movement of the makers, as shown in Fig. 3a. A skeleton-based hand template is created within the motion capture software (Fig. 3b).

\subsection{The design of hand gesture set}

The criteria for our hand gesture set design are that, first the hand gesture set should be natural and easy to use for designers, requiring no special training. Secondly, the hand gesture set should be suitable for continuous recognition in real time. With reference to American Sign Language (ASL) and some previous work $[15,1,18]$, we studied the minimum set of commands that are necessary for 3D conceptual design based on CSG, and proposed the hand gesture set. Recognition of hand gestures involves 1) static hand gesture: static finger configuration without hand movement and 2) dynamic hand gesture: dynamic hand movement with or without finger motion. In comparison with static hand gesture (posture), dynamic hand gesture recognition is more difficult, especially in real time. This is mainly due to the difficulties in determining the start and end points of a meaningful gesture pattern from a continuous stream of input signals. In order to solve this problem, each hand gesture specified for 3D design in our system includes two parts: the posture of the left hand and the dynamic gesture of the right hand. The left hand indicates the type of operation that the designer wants to take, and the right hand specifies the exact action. The user keeps his/her left hand posture unchanged during one operation, i.e., the start and end of the designer's left hand posture indicates the corresponding start and end time of a dynamic action gesture on the right hand. Through this mechanism, the hand gesture recognition system can easily segment the meaningful gestures from 
the continuous input signals which are 3D coordinates of markers on both hands in our motion capture system.

According to the discussion above, the gesture for 3D design can be presented in the following form:

HG3D :=< Type Posture, Action Gesture $>=<T P, A G>$

HG3D stands for the Hand Gesture for 3D design. TP is the posture of the left hand, because this posture indicates the type of operation that the designer wants to take, so we call it type posture. AG (action gesture) is the exact operation performed by the right hand. This form of representation not only makes the structure of a 3D design gesture clear, but can also reveal an important characteristic which will be used in the real time hand gesture recognition. Similar to a $\langle k e y$, value $>$ map structure, TP can also be used as an index to search the hand gesture model database and accelerate the recognition speed.

A meaningful type posture consists of certain palm orientation and the corresponding static finger configuration, as stated in Eq. (2).

$\mathrm{TP}:=<$ Palm Orientation, Finger Configuration $>=<P O, F C>$

In theory, type posture is static, which implies that the designer should not move the left hand and fingers during one operation. However, it is usually difficult for designers to remain still. Therefore, our system allows small, natural movement of left hand, and this movement is ignored when recognizing the left hand gesture.

In contrast to type posture, action gesture defines detailed design operation. Action gesture consists of palm orientation, finger configuration and hand movement of right hand, as stated in Eq. (3).

AG $:=<$ Palm Orientation, Finger Configuration, Hand Movement > $=<P O, F C, H M>$ 
There are two categories of action gestures. In category one action gestures, the trajectory of right hand (precisely, the index finger tip) will form the designed geometry. This category of action gesture is used for creating 2D primitives, which are the basis for $3 \mathrm{D}$ solid generation. The type posture for this operation is to open the left hand and keep all fingers together with the hand palm facing the screen, as shown in Fig. 4a. The left hand represents a 2D plane, and the designer can draw a 2D shape using his/her right index finger (Fig. 4b) while keeping this type posture. The system will know that the designer is drawing a 2D geometric figure and recognize it according to its trajectory.

There are several primitive geometric figures that the designer can draw using his/her right index finger in the system: square, triangle, circle, trapezoid, etc. Then by extruding a 2D shape along a path, i. e., along the $\mathrm{X}$ axis in Fig. 4c, the designer can create some basic solids, such as box, cylinder, wedge, and so on. For the 3D shape which has a geometric central axis, such as sphere, cone or torus, revolving certain 2D geometry around a central axis (Fig. 4d) is the better way to create it. We did not use hand gestures to directly create certain 3D solids, because that requires the designer to remember too many design gestures (one gesture for each 3D primitive solid).

All other action gestures belong to the second category. Some of these action gestures have no hand movement at all, such as action gestures for Union, Subtract, Intersect, Select, Redo, Undo, Copy and Past. Some of these action gestures have one simple hand movement perpendicular to the hand palm, to represent the direction of operation, such as Extrude, Zoom in/out, Scale, Translate and Rotate. The main concern for designing these action gestures is for them to be easily recognisable in real time. The complete set of all the hand gestures in our system, including type postures (TP) of the left hand and the action gestures (AG) of right hand, are shown in Fig. 4, Table 1 and Table 2.

All the hand gesture commands provided by the system are summarised in Fig. 5 according to their functionalities. These hand gesture commands can be classified into two main categories: 3D object operations and workspace operations. 3D object operations involve common functions for 3D design, such as creating, manipulating, and merging solids, while workspace operations just involve six frequently-used operations: undo, redo, copy, paste, zoom in and zoom out. The design of the 
hand gesture set is also aimed at making the system as simple and tidy as possible, in order to facilitate real time hand gesture recognition.

\subsection{Skeleton-based hand model}

Choosing a proper hand model is the basis for efficient hand gesture recognition, because the hand model determines not only the features required for the recognition algorithm, but also the setup of hand motion capture. A skeleton-based model of the human hand [19, 20] is used in our system, as shown in Figure 6. In this type of hand models, two sets of parameters are frequently used for the gesture recognition - angular (joint angles) and linear (phalange lengths and palm dimensions). In our system, the former type of parameters is used as the main features to determine the finger configuration, mainly because the latter one is varying for different people, and in contrast, joint angles vary little between different users for the same gesture. Besides joint angles, the distances between neighbouring fingertips are also evaluated in order to distinguish the predefined gestures from those unmeaning ones, and to enhance the recognition capability of our system.

As the part of a skeleton-based model, some relationships between the movements in neighbouring finger joints are used to reduce the number of hand gesture parameters needed to collect. Rijpkema and Girard [21] proposed a widely used constraint, as stated by Eq. (4).

$$
\theta_{P I P}=\frac{3}{2} \theta_{D I P}
$$

Where $\theta_{\text {PIP }}$ and $\theta_{\text {DIP }}$ represent the flexion DOF for the PIP (Proximal Interphalageal) and DIP (Distal Interphalangeal) joints of each finger. This is a strong constraint and is adopted by many researchers working on hand gesture analysis. According to this constraint, the angle of the DIP joint of each finger does not need to be captured if the angle of the corresponding PIP joint is available.

\section{Calculation}

During a design process, the positions of hand markers are captured and recorded via the software EVaRT 5.0. Based on the event-driven-architecture (EDA) and the functionalities provided by EVaRT 5.0 SDK, the motion data is captured and processed in real time. The captured motion data is immediately processed through a second order Butterworth low-pass filter with a cut-off 
frequency of $5 \mathrm{~Hz}$ before the feature extraction to eliminate the noise. Real time hand gesture recognition is the key for the successful implementation of the rapid 3D conceptual design system. Basically, two mechanisms for real time recognition are employed in the system, template matching and hidden Markov model (HMM) [22]. These will be discussed in detail in the following sections.

\subsection{Hand posture recognition}

As the discussion in section 2.3 indicates, the posture is composed of certain palm orientation and the corresponding static finger configuration. The latter is determined by joint angles and distances between neighbouring fingertips which can be evaluated directly from the coordinates of markers. However, the approach to recognize the palm orientation is not so straightforward. In our system three markers, one of which is located at the wrist joint and the other two are located at the MCP (Metacarpophalangeal) joints of the index and little fingers respectively, are used to define the palm orientation, as shown in Fig. 7a.

In a three-dimensional Euclidean space, any three points will form a plane, provided that they are not collinear. The coordinates of three markers, $\mathrm{W}, \mathrm{P}_{1}$ and $\mathrm{P}_{2}$ should be sufficient to define the palm plane. However, the mathematical definition of a plane through three points is determined by the coordinate values of three points, and planes with the same orientation but different location will result in different mathematical expressions, and thus it is unsuitable to be directly used for real time hand gesture recognition. This is because in hand gesture recognition we are only interested in the palm plane orientation, and the palm plane location is of no interest to us, and can not be stored in the database. Secondly, the palm plane orientation needs to be converted form continuous values to discrete data in order to meet the requirement of real time template matching. Therefore, we developed a new method to evaluate the palm orientation in real time, which includes three steps:

(1) Obtain two vectors from the given three points: $\overrightarrow{\mathrm{WP}_{1}}$ and $\overrightarrow{\mathrm{P}_{1} \mathrm{P}_{2}}$ (Fig. 7 (a)).

(2) Project the vector $\overrightarrow{P_{1} P_{2}}$ onto the xy-plane, then find the angle between this projection and $x$ axis, and denoted it as $\theta_{\mathrm{xy}} \cdot \theta_{\mathrm{xy}}$ is measured from the positive direction of $\mathrm{x}$-axis to the positive direction of projected vector, and lies within the range $0 \leq \theta_{\mathrm{xy}}<2 \pi$. If the vector $\overrightarrow{\mathrm{P}_{1} \mathrm{P}_{2}}$ is 
perpendicular to the xy-plane, the vector $\overrightarrow{\mathrm{WP}_{1}}$ will be used to project onto xy-plane, and the angle between its projection and $\mathrm{x}$-axis will be denoted as $\theta_{\mathrm{xy}}$. Similarly, the vector $\overrightarrow{\mathrm{P}_{1} \mathrm{P}_{2}}$ is projected onto and zx-plane as well, and the angle between the projection and z-axis is denoted as $\boldsymbol{\theta}_{\mathbf{z x}}$, whilst the vector $\overrightarrow{\mathrm{WP}_{1}}$ is projected onto yz-plane and the angle between the projection and y-axis is denoted as $\theta_{\mathrm{yz}}$.

(3) Quantise the three angles to get the corresponding codewords. $\theta_{\mathrm{xy}}, \theta_{\mathrm{yz}}$ and $\boldsymbol{\theta}_{\mathrm{zx}}$ are quantised by $30^{\circ}$ in order to generate the codewords from 1 to 12 (see Figure $7 \mathrm{~b}$ ). For example, if the angle is between $15^{\circ}$ and $45^{\circ}$, the codeword will be 2 . The reason for choosing $30^{\circ}$ as discrete step is that through experiment, we found that $30^{\circ}\left( \pm 15^{\circ}\right)$ is appropriate as the maximum allowed deviation range for the palm orientation in a specific hand gesture.

Finally, the palm orientation can be represented in the form of a three-dimensional feature vector:

$\mathrm{PO}:=\left[\operatorname{code} \theta_{\mathrm{xy}} \operatorname{code} \theta_{\mathrm{xz}} \operatorname{code} \theta_{\mathrm{yz}}\right]$

Where $\operatorname{code} \theta_{\mathrm{xy}}, \operatorname{code} \theta_{\mathrm{yz}}$ and $\operatorname{code} \theta_{\mathrm{xz}}$ represent the respective codewords of $\theta_{\mathrm{xy}}, \theta_{\mathrm{yz}}$ and $\boldsymbol{\theta}_{\mathbf{z x}}$. This feature vector, together with the parameters of joint angles and distances between neighbouring fingertips, constitutes the simplified skeletal model (also called "features" to be extracted) from hand gesture in real time. This model is compared with the hand gesture model templates in the system database, and template matching is used to recognize the designer's static gestures including type postures (TP) and action gestures (AG).

\subsection{Dynamic gesture recognition}

Although some action gestures (AG) are static, most of them are dynamic. There are two types of dynamic action gestures, simple motion type and complex motion type. In the simple motion type action gestures, the hand motion is always perpendicular to hand palm plane. The hand motion in action gestures for Extrude (Fig. 4c), Zoom in/out (Fig. 4i), Scale, Translate (Table 1) and Rotate (Table 2) belongs to this type. Because the motion of hand is simple and can be easily determined by tracking the position of certain marker such as the one attached to the wrist, this type of dynamic gestures can be easily recognized. The other type, complex motion type dynamic gesture is used to 
create $2 \mathrm{D}$ primitives (Fig. $4 \mathrm{~b}$ ), and requires tracking the trajectory of the fingertip and recognizing which 2D geometric shape the designer is drawing. In order to accomplish this task, a real time recognition algorithm based on HMM is employed.

The trajectory of the fingertip can be represented as a $3 \mathrm{D}$ polyline in time sequence: $\left\{\mathrm{P}_{\mathrm{i}}\right\}, \mathrm{i}=$ $1,2, \ldots, \mathrm{T}$, where $\mathrm{P}_{\mathrm{i}}$ is a $3 \mathrm{D}$ position of the fingertip marker, and the direction of movement is determined by two consecutive points from the movement path (Fig 8). Since we setup the motion capture space with the plane in front of the designer as xy-plane, the fingertip motion in creating 2D primitive action gesture is expected to be in xy-plane. In order to accelerate the recognition process, a $3 \mathrm{D}$ to $2 \mathrm{D}$ reduction is carried out. Given any two consecutive points $\mathrm{P}_{\mathrm{m}}$ and $\mathrm{P}_{\mathrm{m}+1}$, the direction of finger movement is determined as follows:

(1) Compute $\delta_{\mathrm{x}}=\mathrm{X}_{\mathrm{m}+1}-\mathrm{X}_{\mathrm{m}}, \delta_{\mathrm{y}}=\mathrm{Y}_{\mathrm{m}+1}-\mathrm{Y}_{\mathrm{m}}, \delta_{\mathrm{z}}=\mathrm{Z}_{\mathrm{m}+1}-\mathrm{Z}_{\mathrm{m}}$ and find $\delta_{\max }=$ $\max \left(\left|\delta_{\mathrm{x}}\right|,\left|\delta_{\mathrm{y}}\right|,\left|\delta_{\mathrm{z}}\right|\right)$

(2) Calculate $(\mathrm{dx}, \mathrm{dy}, \mathrm{dz})=\left(\delta_{\mathrm{x}} / \delta_{\max }, \delta_{\mathrm{y}} / \delta_{\max }, \delta_{\mathrm{z}} / \delta_{\max }\right)$ and find out which one is closest to zero, then assign it to zero (implies that its dimension is zero). The other two components will constitute a plane to replace the 3D space. For example, if $\mathrm{dz}$ is zero, $\mathrm{xy}$-plane will replace the 3D space.

(3) Project the vector $\overrightarrow{\mathrm{P}_{\mathrm{m}} \mathrm{P}_{\mathrm{m}+1}}$ onto that plane and calculate the angle between the horizontal axis and this projection. Taking $\mathrm{dz}=0$ as an example, the angle is given by $\theta=\arctan \left(\delta_{\mathrm{y}} /\right.$ $\left.\delta_{\mathrm{x}}\right)$

(4) Quantize the angle at an interval of $20^{\circ}$ in order to generate the codewords from 1 to 18 (similar to Fig. 7b).

A codeword of the direction can be obtained for each frame sampled for gesture recognition (except the first frame) from the motion data. For a hand gesture sampled for $\mathrm{T}$ fames, a $(\mathrm{T}-1)$ dimensional feature vector is obtained and used as input to HMM.

In the system database there are discrete HMMs corresponding to each $2 \mathrm{D}$ primitives. According to the experimental results of some previous researchers [23, 24], Left-Right Banded (LRB) model 
structure (Figure 9) performed better than other HMM topologies for dynamic hand gesture recognition in respect to the recognition rate and computation consumption. Therefore, LRB HMMs with different number of states are used to model the finger motion in the system. Basically, there is no fixed value for the number of states for all HMMs, and the number of states is determined individually based on the complexity of each dynamic hand gesture trajectory to generate 2D primitive. The number of states for each HMM is determined by experiment, and the criteria is to have the minimum number of states to robustly distinguish a trajectory. This is because the excessive number of states can generate the over-fitting problem and more training samples are needed. If the number of states is insufficient, the discrimination power of the HMM will be reduced since at least one state should be modelled for every geometric features in the $2 \mathrm{D}$ primitive trajectory.

All these initial HMMs are trained by utilizing the Baum-Welch algorithm [25]. In order to guarantee that a full training is completed, sufficient training samples (more than 20 for each gesture in our system) are captured and provided. Afterwards, all trained HMMs are organized into an HMM network (Fig. 10).

The real-time dynamic hand gesture recognition is implemented by using the Forward-Backward algorithm which evaluates the probability $P(0 \mid \lambda)$ that the observation sequence 0 was generated by the given model $\lambda$. Each feature vector of finger movement, as an observation sequence, is extracted in real time, and used as the input to the HMM network (Fig. 10). $P(0 \mid \lambda)$ is then calculated in parallel for all gesture HMMs in the network by employing the Forward-Backward algorithm [24]. If the $P(0 \mid \lambda)$ value for $i^{\prime}$ th model is the maximum, then the sequence will be classified into class $i$. Because in this algorithm, a model with the maximum $P(O \mid \lambda)$ will be picked no matter what the input is, there is a possibility that the input gesture is unmeaningful or unrelated to the current design scenario. In order to correct this problem, a method to provide an adaptive threshold for classification of meaningful gestures and unrelated finger movements using HMM, as what Lee and Kim [26] proposed, is also utilized in the system. A hand gesture will be recognised only if it is with the maximum $\mathrm{P}(\mathrm{O} \mid \lambda)$ and above the threshold of a HMM. Otherwise, it will be classified as an unrecognised gesture. 


\subsection{Hand gesture model database}

According to Eq. (1), each hand gesture for 3D concept design includes two parts: type posture of left hand and action gesture of right hand. Using the methods discussed in previous sections, both type posture and action gesture are represented in terms of skeleton model based templates or HMMs. For each hand gesture defined in the system, corresponding models of different templates and HMMS are created in advance. In order to get the best recognition performance, specifically in real time, all these models are organized into a tree structure (Fig. 11) in the system database. There are two advantages for this structure: (1) the same template can be shared between different hand gestures. Shared templates can not only reduce the size of the whole database, but also reduce the complexity of adding new hand gestures later on. (2) There is no recursive or iterative process during the searching in the tree structure we defined. If no child node can be reached from a non-leaf node, the search process stops, and the input hand gesture is declared to be unmeaningful. The search process is only forward, every step from one node to its child node can reduce the search space significantly because the rest branches from that hierarchy will not be searched. This makes the search process most efficient and accelerate the recognition speed.

\section{Results and Discussion}

\subsection{System UI and Implementation}

According to the theory of distributed cognition, external information presented in an appropriate format can reduce the difficulty of a task by supporting recognition-based memory or perceptual judgements rather than recall [27, 28]. For traditional WIMP (Windows-Icons-Menus-Pointers) interfaces, one of the advantages is that some icons are self-explanatory so that users do not have to remember lots of commands. However, for most hand gesture based systems, there are no specially designed self-explanatory user interfaces proposed, so users need a long training time to remember a large set of hand gestures before they can use the system in practice. In order to solve this problem, an rule-based intelligent user interface was designed in our system. The basic idea is that the system gives users a list of alternative hand gestures which can be performed next step after an operation has been completed. These alternative hand gestures are also graphically illustrated and prompted on the screen as reminders, so that users do not have to remember them. The selection of alternative hand 
gestures is based on a set of rules taking into consideration the different design tasks users are currently performing, as well as the current state of the workspace.

- Rule 1: if there is only one 3D object in the workspace, the gestures to be performed on two objects, such as Boolean operations, are not listed as alternatives.

- Rule 2: if there are two 3D solids in the workspace, and no one has been selected, all manipulation gestures except the 'Select' operation are not listed as alternatives; in contrary, if one 3D solid has been selected, all manipulation gestures except the 'select' operation are listed as alternatives.

- Rule 3: when the designers perform a type posture, all action gestures working in pair with this type posture, are listed as alternatives. If some of them are against the Rule 1, they will be eliminated.

- Rule 4: according to the associative relationships among different hand gestures, which are summarized form common design experience, some gesture commands are frequently followed by a certain set of other gestures. Drawing a 2D primitive, for example, is most likely followed by extruding or revolving it to create a 3D shape. The likelihood of next operation is used to sort the alternatives, with the most likely on the top of the alternative list.

- Rule 5: Workspace operations, including undo, redo, copy and paste, are always as part of the alternative list.

An example of the prototype system UI is shown in Figure 12. On the left side window, it is the open source OpenSCAD [29], which is employed as the workspace to display the designed 3D objects. We did not make any alterations to the OpenSCAD environment, and only use it as the displaying window. In the current example, it is activated and the designed chair seat panel is selected (in red). On the right side widow, it is the user interface we implemented. On the top of the window, the hand gesture recognition result is displayed, and in current example, it is 'Translate' along ' $x$ axis'. In the middle of the window, all the alternatives are listed. In the bottom of the window, the connection status with motion capture system is displayed.

The prototype system is implemented with Visual C++ and MATLAB. The core recognition algorithms were written in MATLAB, then compiled to C++ DLL. All other parts in the system, 
were programmed with Visual $\mathrm{C}++$. The recognised hand gestures are translated into OpenSCAD script language, and then displayed in OpenSCAD window. The 3D geometry generation in our system is accomplished in OpenSCAD, which supports constructive solid geometry. Fig. 13 shows the system implementation.

\subsection{A case study: designing a chair}

We tested our hand gesture based rapid 3D conceptual design method with the task of designing a chair. A small number of undergraduate students, research students performed as designers to carry out the design task. An example of the design process is as following:

(1) Draw a circle and extrude it to create a cylinder, then repeat to create another cylinder with different radius and height, then perform the subtract operation to obtain a toroid, as show in Fig. 14a.

(2) Draw a square and extrude it to create a cube, and then make it intersect with the toroid, as shown in Fig. 14b. After performing the intersect operation, the back of the chair is obtained (Fig. 14c).

(3) Draw a square and extrude it to create a cube, scale it (Fig. 14d), which is used as the chair seat panel.

(4) Union the back of the chair and the chair seat panel together, as shown in Fig.14e.

(5) Draw a circle and extrude it to create a cylinder, and scale it to appropriate size for a chair leg (Fig. 14f).

(6) Copy this chair leg, for later to duplicate

(7) Move this chair leg to the proper position and perform the union operation.

(8) Paste to generate a duplicated chair leg and move it to the proper position and perform the union operation.

(9) Repeat Step (8 ) for 2 times to create and position other chair legs. The final chair model is shown in Fig. 14g.

In our test, the cameras were set to 120 frames per second. The designers spent less than 10 min to complete the design modelling task after a brief introduction to the system. More chairs created by designers in this experiment are shown in Fig. 15. However, creating the 3D model from scratch every time is sometimes unnecessary and time-consuming. To facilitate the usage of our hand 
gesture based conceptual design system, some non primitive solids, which are generated from primitive solids by using Boolean operations, are provided as a library and can be browsed and picked up and used directly by designers (Fig. 16). Through selecting, manipulating and merging these solids provided by the library, designers can create many conceptual models rapidly just like playing Lego.

\subsection{Discussion}

The 3D shape modelling method in our proposed system is a hand gesture-based procedural method, and the instantly generated product model is represented by OpenSCAD command scripts in the form of a sequence of commands used to create the product shape and topology, which is very similar to the neutral macro file proposed by Cheon et al [30]. The advantage of the procedural method is that it can capture the design intent, model construction history, features and constraints [31], which are very important aspects in conceptual design stage. That is why Cheon et al [30] identified the need to transfer the boundary representation (B-rep) data obtained from 2D sketch into procedural model. From this point of view, our proposed system is suitable for concept design.

For the purpose of 3D styling in conceptual design, 3D sketch with a digitized pen is another optional method. We have used the 3D sketch tool 'Leonar3Do' to support structural design at the conceptual design stage [32], and found that a 3D digitalized pen can either provide raw and pure geometric data for a reversing engineering process to generate a smoothed $3 \mathrm{D}$ model or provide a set of gesture information. In the latter case, it is similar to the proposed system in the current paper, but the designer will typically operate in one-hand. With the proposed system in the current paper, the designer will be free from device-induced constraints and use gestures from two hands along with some body movements to express their 3D design intentions. The accuracy of position information from the motion capture system is better but the equipment is expensive. The modelling speed is depending on the reliability of gesture recognition for both systems. In terms of easiness, the system uses two-hands is generally considered to be easier by the user.

We defined a hand gesture set which contains both left hand gesture and right hand gesture, and they are collectively called HG3D. The left hand gesture is static without hand and finger movement, and it is called TP (type posture). TP is used to indicate the category of a specific hand gesture, i. e., which group (Fig. 11) this hand gesture is belong to. The right hand gesture is dynamic 
with hand and finger movement, and it is called AG (action gesture). HG3D contains TP and AG (Equation 1). For example, Fig 4a is a TP which means now creating 2D primitives, whilst Fig 4b is an $\mathrm{AG}$ which can generate various $2 \mathrm{D}$ primitives based on the sketch (trajectory) of index finger. In the 16 examples in Fig 4, if the illustrated hand gesture is on one hand, it is designated as TP (left hand) or AG (right hand). If the illustrated hand gesture is on both hands, it is designated as HG3D. By defining a hand gesture set with TP and AG, hand gesture recognition error has been minimised, because TP defined the hand gesture category, and within this category, there are only a few different hand gestures.

The system is currently still in the prototype and development stage, and we are trying to use different algorithms to optimise the system. Different ready-made MATLAB algorithms can be easily hooked up with $\mathrm{C}++$ to try out. Once the optimisation process is completed, the entire system will be implemented with C++ only.

\section{Conclusion and future work}

A new method of using hand gesture and motion capture system as user interface for rapid 3D conceptual design in real time has been systematically investigated and tested, and a prototype system has been implemented. The hand gesture set proposed is simple, natural and kept to minimum number, and no need for training and tedious remembering. Each hand gesture consists of two parts: type posture of left hand and action gesture of right hand. The type posture not only indicates the type of operation, but also used to segment the starting and ending time for the action gesture of right hand from the continuous input signals. Some simplification and quantisation techniques are employed in the real time gesture recognition algorithms, such as codewords to represent the palm orientation and finger trajectory, wrist marker to represent hand movement, PIP angle and fingertip marker distance as features for hand posture. Experiment results shows that these algorithms are robust and meet the requirement for real time recognition. The rule-based intelligent user interface changes the icons instantly according to design process and expected operations, and prompt the alternative had gestures graphically. The recognised hand gestures are translated into OpenSCAD script language, and instantly displayed in WYSIWYG style. 
The significance of the current research is that it realised using hand gestures only to complete the entire $3 \mathrm{D}$ conceptual design in real time for the first time. Although the prototype system is very simple, and there are lots of rooms for extension and further improvement, it has revealed the potential of using hand gestures as a new user interface for rapid 3D conceptual design. Currently, there is a new trend of using hand gestures to interact with computers and to control domestic appliances. The principles and techniques reported here can be used for other applications as well, and they are not restricted to motion capture system and can be adopted in video image based system as well.

For future work, we will seek alternative algorithms for the recognition of hand gesture trajectory which require less training than HMMs. We will also extend the system to be able to handle multiple 3D components and assemble them, and incorporate the standard part library, in order to design complicated products.

\section{References}

[1] Zheng, J. M., Chan, K. W., and Gibson, I. 2001. Desktop virtual reality interface for computer aided conceptual design using geometric techniques. J. Engineering Design, 12(4), 309-329.

[2] Robertson B, Radcliffe D. 2009. Impact of cad tools on creative problem solving in engineering design. Computer-Aided Design, 41(3):136-46.

[3] Sachs, E., Roberts, A., and Stoops, D. 1991. 3-Draw: A tool for designing 3D shapes. IEEE Computer Graphics and Applications, 11(6), 18-26.

[4] Chen, Y., Liu, J., and Tang, X. 2008. Sketching in the Air: A Vision-Based System for 3D Object Design. IEEE Conference on Computer Vision and Pattern Recognition (CVPR), pp. 1-6.

[5] Vinayak, Murugappan, S., Liu, H., Ramani, K., 2013. Shape-It-Up: Hand gesture based creative expression of 3D shapes using intelligent generalized cylinders. Computer-Aided Design, 45(2), 277-287.

[6] Chapin, W. L., Lacey, T. A., and Leifer, L. 1994. DesignSpace: A Manual Interaction Environment for Computer Aided Design, Proceedings of the SIGCHI Conference on Human Factors in Computing Systems, Boston, Massachusetts, United States, pp. 33-34.

[7] Chu, C-C. P., Dani, T. H., and Gadh, R. 1997. Multi-sensory user interface for a virtual reality based computer aided design system. Computer Aided Design, 29(10), 709-725.

[8] Chu, C-C. P., Dani, T. H., and Gadh, R. 1998. Evaluation of virtual reality interface for product shape designs. IIE Transactions, 30(7), 629-643.

[9] Pouliquen, M., Bernard, A., Marsot, J., Chodorge, L., 2007. Virtual hands and virtual reality multimodal platform to design safer industrial systems. Computers in Industry,58(1), 46-56

[10] Fuge, M., Yumer, M.E., Orbay,G., Kara, L.B., 2012. Conceptual design and modification of freeform surfaces using dual shape representations in augmented reality environments. Computer-Aided Design 44 (10), 1020-1032.

[11] De Arau'jo, B.R., Casiez, G., Jorge, J.A., Hachet,M., 2012. Mockup builder: 3D modeling on and above the surface, Computers \& Graphics, http://dx.doi.org/10.1016/j.cag.2012.12.005 
[12] O'Hagan, R. G., Zelinsky, A., and Rougeaux, S. 2002. Visual gesture interface for virtual environments. Interacting with Computers, 14(3), 231-250.

[13] Erol, A., Bebis, G., Nicolescu, M., Boyle, R. D., and Twombly, X. 2007. Vision-based hand pose estimation: A review. Computer Vision and Image Understanding, 108(1-2), 52-73.

[14] Figueiredo, M., Bohm, K., and Teixeira, J. 1993. Advanced interaction techniques in virtual environments. Computers and Graphics, 17(6), 655-661.

[15] Holz C, Wilson A., 2011. Data miming: inferring spatial object descriptions from human gesture. In: Proceedings of the 2011 annual conference on Human factors in computing systems. New York (NY, USA): ACM; 811-820.

[16] Condell, J., Moore, G., 2009. HandPuppet3D: Motion capture and analysis for character animation. Artificial Intelligence Review. 31(1-4),45-59.

[17] Mapes, D. P., and Moshell, J. M. 1995. A two-handed interface for object manipulation in a virtual environment. Presence: Teleoperators and Virtual Environments, 4(4), 403-416.

[18] Wu, Y., and Huang, T. S. 1999. Vision-Based Gesture Recognition: A Review. Lecture Notes in Computer Science, Vol. 1739, 103-115.

[19] Mitra, S., and Acharya, T. 2007. Gesture Recognition: A Survey. IEEE Trans. Systems, Man, and Cybernetics, Part C: Applications and Reviews, 37(3), 311-324.

[20] Wang, R. Y. and Popovic, J. 2009. Real-Time Hand-Tracking with a Color Glove, ACM Transaction on Graphics (SIGGRAPH 2009), 28(3), August 2009.

[21] Shih, N-J. and Shih, W-D. 1996. Gesture modelling for architectural design. Computers \& Graphics, 20(6), 849-862.

[22] Qin, S., Wright, D. K., Kang, J., and Prieto, P. A. 2006. Use of 3D body motion to freeform surface design, Proceedings of IMechE. Part B. J. Engineering Manufacture, 220(2), 335339.

[23] Qin, S. F., Prieto, P. A., and Wright, D. K. 2008. A novel form design and CAD modelling approach. Computers in Industry, 59(4), 364-369.

[24] Yi, X., Qin, S., and Kang, J. 2009. Generating 3D architectural models based on hand motion and gesture. Computers in Industry, 60(9), 677-685.

[19] Lee, J., and Knuii, T. L. 1995. Model-based analysis of hand posture. IEEE Computer Graphics and Applications, 15(5), 77-86.

[20] Pavlovic, V. I., Sharma, R., and Huang, T. S. 1997. Visual Interpretation of Hand Gestures for Human-Computer Interaction: A Review. IEEE Trans. Pattern Analysis and Machine Intelligent, 19(7), 677-694.

[21] Rijpkema, H., and Girard, M. 1991. Computer animation of knowledge-based human grasping. ACM SIGGRAPH Computer Graphics, 25(4), 339-348.

[22] Rabiner, L. R. 1989. A tutorial on hidden Markov models and selected applications in speech recognition. Proceedings of the IEEE, 77(2), 257-285.

[23] Liu, N., Lovell, B. C., Kootsookos, P. J., and Davis, R. I. A. 2004. Understanding HMM Training for Video Gesture Recognition. Proceedings of the Analog and Digital Techniques in Electrical Engineering (TENCON), Chiang Mai, Thailand, pp. 567-570.

[24] Elmenzain, M., Al-Hamadi, A., Appenrodt, J., and Mihaelis, B. 2009. A hidden Markov modelbased isolated and meaningful hand gesture recognition. International Journal of Electrical, Computer, and System Engineering, 3(3), 156-163.

[25] Rabiner, L. R., and Juang, B. H. 1993. Fundamentals of Speech Recognition. Prentice Hall, New Jersy.

[26] Lee, H., and Kim, J. H. 1999. An HMM-Based Threshold Model Approach for Gesture Recognition, IEEE Trans. Pattern Analysis and Machine Intelligence, 21(10), 961-973.

[27] Zhang, J. 1991. The interaction of internal and external representations in a problem solving task. Proceedings of the Thirteenth Annual Conference of Cognitive Science Society, Hillsdale, NJ: Erlbaum.

[28] Hollan, J., Hutchins, E., and Kirsh, D. 2000. Distributed cognition: Toward a new foundation for human-computer interaction research. ACM Transactions on Computer-Human Interaction, 7(2), 174-196. 
[29] OpenSCAD 2011. OpenSCAD User Manual, Available from:

http://en.wikibooks.org/wiki/OpenSCAD User_Manual.

[30] Cheon, S.U., Kim, B.C., Mun, D., Han, S., 2012. A procedural method to exchange editable 3D data from a free-hand 2D sketch modeling system into 3D mechanical CAD systems, Computer-Aided Design, 44(2), 123-131.

[31] Kim, J., Pratt, M. J., Iyer, R.G., Sriram, R.D., 2008. Standardized data exchange of CAD models with design intent. Computer-Aided Design, 40(7):760-77.

[32] Prieto, P. A., Soto, F. D., Zúñiga, M. D., Qin, S., Wight, D. K., 2012. Threedimensional immersive mixed reality interface for structural design, Proc IMechE Part B: J Engineering Manufacture, 226(5) 955-958. 